\title{
La zaratita de Cabo Ortegal (A Coruña): historia de su descubrimiento y caracterización actual
}

\author{
The zaratite of Cape Ortegal (A Coruña): discovering history and \\ current characterization
}

\author{
A. La Iglesia1, J. Garcia-Guinea², J. González del Tánago³ \\ 1 Instituto de Geociencias (CSIC, UCM). José Antonio Novais, 2. 28040 Madrid, Spain. Email: iglesia@ucm.es \\ 2 Museo Nacional de Ciencias Naturales (CSIC). Jose Gutiérrez Abascal, 2. 28006 Madrid, Spain \\ 3 Departamento Petrología y Geoquímica Facultad de Ciencias Geológicas, Universidad Complutense 28040 Madrid, Spain
}

\section{RESUMEN}

La zaratita, $\mathrm{Ni}_{3} \mathrm{CO}_{3}(\mathrm{OH})_{4} \cdot 4 \mathrm{H}_{2} \mathrm{O}$ es uno de los pocos minerales descubiertos en España. Por ser una fase de baja cristalinidad y presentar una composición química variable según los diferentes yacimientos, la International Mineralogical Association (IMA) actualmente la considera con status Q (questioned). En este artículo se analizan en primer lugar los descubrimientos, casi simultáneos, del mineral español y del llamado "Emerald of Nickel" de Texas, Lancaster County, Pennsilvania (USA), de similar composición química, que dieron lugar a la actual zaratita, donde el mineral español aportó el nombre y el americano la composición química y la descripción de las propiedades físicas. En segundo lugar, se presenta una nueva caracterización de la muestra histórica de zaratita de Cabo Ortegal, por microsonda electrónica, microscopia electrónica de barrido, difracción de rayos-X, análisis termo-gravimétricos y termo-diferenciales, micro-Raman, espectroscopia infrarroja y catodoluminiscencia espectral y se discuten los resultados analíticos.

Palabras Clave: zaratita; níquel; carbonato; Cabo Ortegal; A Coruña

\begin{abstract}
Zaratite, $\mathrm{Ni}_{3} \mathrm{CO}_{3}(\mathrm{OH})_{4} \cdot 4 \mathrm{H}_{2} \mathrm{O}$ is one of the few minerals discovered in Spain. Taking into account that it is an amorphous compound with variable chemical composition depending on the ore deposits, the International Mineralogical Association (IMA) currently considers zaratita in the Q status (Questionable). In this article we analyze first the nearly simultaneous discoveries of the Spanish zaratite and other material called "Emerald of Nickel" from Texas, Lancaster County, Pennsylvania (USA), of similar chemical composition. Both led to the current zaratite, where the Spanish mineral provides the name and the American one, the chemical composition anc physical properties. In addition, here we also include an experimental characterization of the zaratite historical specimen from Ortegal Cape, by electron microprobe, scanning electron microscopy, X-ray diffraction, differential thermal and thermo-gravimetric analyses, micro-Raman, infrared transmission spectroscopy and spectral cathodoluminescence together with a discussion of the analytical results.
\end{abstract}

Keywords: zaratite; nickel; carbonate; Cape Ortegal; A Coruña

Recibido: 20 de marzo de 2013 / Aceptado: 25 de junio de 2013 / Publicado: 18 de junio de 2014

Citation I Cómo citar este artículo: A. La Iglesia, et al. (2014). La zaratita de Cabo Ortegal (A Coruña): historia de su descubrimiento y caracterización con técnicas analíticas no destructivas. Estudios Geológicos, 70(1): e003. doi: http://dx.doi.org/10.3989/egeol.41353.275

Copyright: (c) 2014 CSIC. This is an open-access article distributed under the terms of the Creative Commons Attribution-Non Commercial (by-nc) Spain 3.0 License. 


\section{Introducción}

La zaratita, $\mathrm{Ni}_{3} \mathrm{CO}_{3}(\mathrm{OH})_{4} \cdot 4 \mathrm{H}_{2} \mathrm{O}$, ha sido un mineral dudoso desde su descubrimiento hasta nuestros días. Al tratarse de una fase de baja cristalinidad, la caracterización original fue dificultosa; la composición química varía según la procedencia de las muestras y en difracción de rayos X se obtienen diagramas difusos, con bandas de baja intensidad, que también son diferentes para las distintas muestras. Debido a estas circunstancias, actualmente, el mineral está considerado con status $\mathrm{Q}$ (questioned) por la International Mineralogical Association (IMA). Fue descubierto hacia 1845-1850 por el Prof. Casares junto con la morenosita en los acantilados del Cabo Ortegal, ubicación considerada como Localidad Tipo para ambas especies. Actualmente no se encuentra ninguno de estos minerales ni en el Cabo Ortegal, ni en sus inmediaciones. Los únicos ejemplares españoles de zaratita disponibles son los depositados en el Museo de Ciencias Naturales de Madrid (Fig 1) y en el Museo de Historia Natural de la Universidad de Santiago de Compostela. El estudio de esta especie tiene un gran interés científico por dos razones; en primer lugar, por tratarse de uno de los 30 minerales descubiertos en España y en segundo lugar, por ser uno de los pocos minerales encontrados por un científico autóctono. Recientes estudios por microsonda electrónica (MSE), microscopia electrónica de barrido ambiental con análisis por espectroscopia de energías dispersivas de rayos X (MEBA-EDS), difracción de rayos-X (DRX), análisis termo-gravimétricos y termo-diferenciales (ATD-TG), micro-raman (Raman), espectroscopia infrarroja de transmisión por transformada de Fourier (IRTF) y cátodo-luminiscencia espectral espacialmente resuelta $(\mathrm{CL})$ han arrojado nueva luz sobre la caracterización de la zaratita original. En este artículo presentamos nuevos resultados analíticos junto con una revisión de la historia del descubrimiento del mineral. Debido a la extrema escasez de muestra disponible, las distintas determinaciones se han realizado utilizando técnicas no destructivas como la microscopia electrónica ambiental o destructivas en el rango de la micro-muestra, utilizando portamuestras de silicio para la difracción de rayos X. La cantidad total de muestra empleada no excedió de $20 \mathrm{mg}$, por lo que la realización de los distintos espectros ha constituido un gran reto.

\section{Descubrimiento y antigua caracterización de la zaratita}

El descubridor de la zaratita y la morenosita, Antonio Casares Rodríguez (Monforte de Lemos 1812-Santiago de Compostela 1888) fue un insigne químico y farmacéutico. Primer titular de la Cátedra de Química de la Universidad de Santiago en 1845 y Rector de esa universidad desde 1872 a 1888 . Está considerado como el padre de la Química Analítica española e iniciador de la espectroscopía en España. En 1851 el Profesor Casares remitió una memoria a la Real Academia de Ciencias donde describía y caracterizaba a dos nuevos minerales de níquel: morenosita y zaratita. En la actualidad, en los archivos de la Academia no aparece esta memoria, pero sí una nota fechada el 8 abril 1851, del Académico Sr. Moreno al Secretario de la Academia en la que presenta la memoria de Casares. Por su interés se recoge a continuación el contenido de la nota:

"El Sr. D. Antonio Casares Catedrático de Química de la Universidad de Santiago y socio corresponsal de la Real Academia de Ciencias me remite para presentar a la misma varios ejemplares de dos especies nuevas de níquel que ha descubierto, acompañando al mismo tiempo una pequeña memoria o nota de su descripción y análisis.

Tengo la mayor satisfacción de desempeñar este cometido dirigiéndome a V. para que se sirva hacerlo presente a la Academia, a cuyo fin le remito también los ejemplares y nota expresados".

Martínez Alcibar (1851) publica una transcripción de la memoria de Casares a la Academia y de ella tomamos estos párrafos:

"Habiendo tenido ocasión de examinar nuevos ejemplares con alguna detención, el resultado de estos exámenes es, que se hallan en ellas dos nuevas especies de mineral de nickel no descritas aun por los autores y son:

Carbonato de nickel.- Sustancia de color verde esmeralda más ó menos oscuro, lustre craso, alguna vez vítreo; no se presenta cristalizada y si en pequeñas masas recubriendo a un mineral de hierro magnético o en el interior de las masas; se raya con la navaja y el polvo es de color verde manzana. Tratado con acido nítrico se disuelve con mucha lentitud y con efervescencia: la disolución es verde: tratada con acido sulfhidrico da indicios de sulfuro negro de cobre: con el amoniaco toma color azul y se 


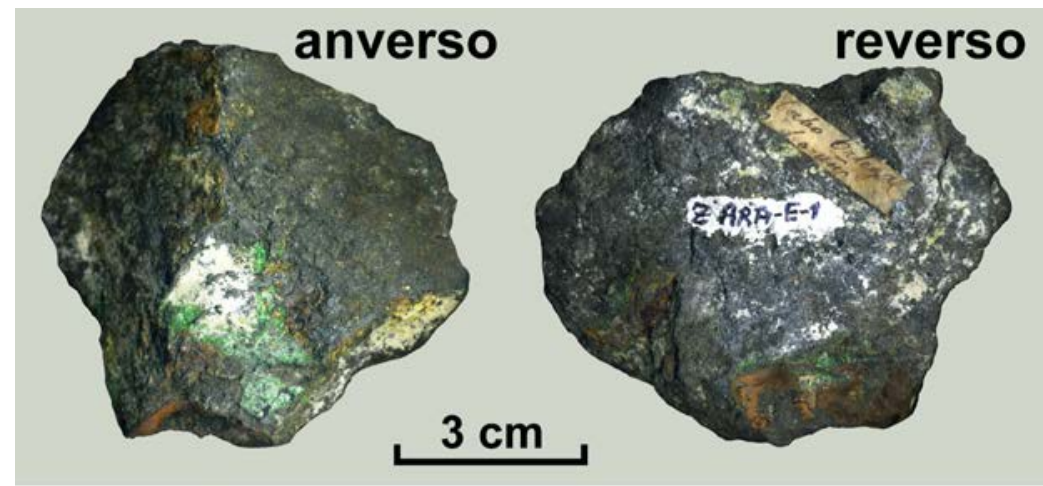

Fig. 1.-Ejemplar de zaratita del Museo Nacional de Ciencias Naturales de Madrid.

precipita una muy pequeña cantidad de oxido férrico.-Al soplete se ennegrece pero no funde; calentándola fuertemente con sosa y un poco de bórax se obtienen a veces globulitos metálicos brillantes de color amarillo claro, magnéticos y que tienen los caracteres del nickel. Calentando este mineral en un tubo de vidrio cerrado por un extremo da vapores acuosos.- Es por consiguiente el mineral carbonato de nickel hidratado casi puro".

"Sulfato de nickel......."

"En el tratado de mineralogía de Dufrenoy, en las obras de Hoffmann, Haüy y Beudant no se hace mención de estas especies de mineral de nickel, por lo que creo que son enteramente nuevas, y si la Academia me permite darlas nombre, denominaría a la primera zaratita y a la segunda morenosita, dedicándolas a los señores Gil y Zarate y Moreno".

Antonio Gil y Zarate (1796-1861) fue director general de Instrucción Pública y miembro de las Academias de la Lengua y de Bellas Artes (Fig. 2). Antonio Moreno Ruiz (1796-1852), Académico de la de Ciencias Naturales fue precisamente, el que presentó la memoria de Casares. Al Académico Sr. Amar de la Torre (1802-1874) se le encargó estudiar la memoria de Casares y contesta el 28 abril 1851 confirmando la importancia del trabajo y desechando las denominaciones propuestas:

"Evacuando el informe que V.S. se ha servido pedirme acerca de la memoria del Sr. Casares, relativa a unos minerales de carbonato y sulfato de niquelo, debo manifestarle que las noticias que contiene, si bien no forman una característica completa de dichas especies, las juzgo dignas de insertarse en las memorias de la Academia. En cuanto a las denominaciones que propone darles, de ningún modo pertenecen a la nomenclatura científica que se funda en propiedades características, sino a la llamada impropiamente trivial que solo sirve para confusión de las ciencias, y por lo mismo convendría que su autor las remplazase por otras más convenientes."

La opinión de la Academia tuvo poca influencia ya que las nuevas especies fueron conocidas a través de los artículos publicados en 1850 y 1851 por Martínez Alcibar, conservando las denominaciones originales.

En 1847 Silliman publica la descripción de un nuevo mineral de Texas, Lancaster County, Pennsilvania, conocido por los mineralogistas americanos como "green oxide of chrome", incluyendo datos sobre dureza en la escala de Mohs 3-3,25 densidad 2,570$2,693 \mathrm{~g} / \mathrm{cm}^{3}$ lustre, color, raya, etc., asignándole una composición química de oxido de níquel hidratado. Un año mas tarde, en un nuevo artículo sobre el mineral, al que ya denomina "Emerald of nickel", corrige la composición química indicando que se trata de un carbonato en lugar de un óxido y presenta el siguiente análisis químico: óxido de níquel 58.811, acido carbónico $\left(\mathrm{CO}_{2}\right)$ 11,691 y agua 29,498\%.

Smith y Brush (1853) en una "reexamination of American minerals" publican el siguiente análisis para el mineral "Emerald of nickel" de Pennsilvania: óxido de níquel 56,82, magnesia 1,68, acido carbónico 11,63 y agua $29,87 \%$. Con estos valores obtuvieron la siguiente composición: oxido de níquel $3, \mathrm{CO}_{2} 1$, agua 6; es decir: $\mathrm{Ni}_{3} \mathrm{CO}_{3}(\mathrm{OH})_{4} \cdot 4 \mathrm{H}_{2} \mathrm{O}$. En la segunda edición de Dana (1878) el mineral de Pennsilvania ya aparece como descubierto por Silliman, denominado zaratita y con la composición química anterior. Aunque Kiselev (1938) da una composición distinta: $\mathrm{NiCO}_{3} \mathrm{Ni}(\mathrm{OH})_{2} \cdot 3 \mathrm{H}_{2} \mathrm{O}$, la de Silliman continuará apareciendo en todos los tratados de Mineralogía publicados desde esa fecha hasta los mas actuales con las siguientes modificaciones: inclusión 
de Casares como descubridor y al Cabo Ortegal como Localidad Tipo (Palache et al., 1951). En 1963 Isaacs da la voz de alarma sobre la defectuosa caracterización del mineral, al indicar que los datos de las propiedades físicas y químicas de la zaratita varían significativamente según la procedencia del ejemplar, citando análisis de Williams en los que el contenido en elementos oscila entre los siguientes rangos: $\mathrm{NiO}$, desde 56,9 a 61,$2 ; \mathrm{CO}_{2}$, desde 13,5 a 15,$7 ; \mathrm{H}_{2} \mathrm{O}$, desde 23,2 a $27,1 \%$. A partir de entonces todo han sido dudas sobre el mineral.

Los estudios de difracción de rayos $\mathrm{X}$ sobre la zaratita han sido tan controvertidos como su caracterización química; el primero en publicar datos de rayos $\mathrm{X}$ fue Fenoglio (1934a, 1934b) quien estudia varias muestras procedentes de Lancaster County y Lilaz, Valle d'Aosta, Italia. Según este autor, "los espectros obtenidos son netos y perfectamente iguales" y le permiten asignar una celdilla cúbica, "compatible con la isotropía del mineral", con $\mathrm{a}_{0}=6,35 \AA$.

Williams et al. (1959), estudian la "Emerald green mineral" de Heazlewood, Tasmania (descrito por Petterd en 1894 y denominándolo zaratita) un ejemplar con composición química similar al de Lancaster County. Al realizar la difracción de ambas muestras obtienen diagramas difusos, aunque similares, donde no es posible medir espaciados con facilidad "it coud not be indexed on the basis of a cubic cell atributed to zaratite by Fenoglio (1934) and quoted by Palache et al. (1951)". Isaacs (1963) estudia nuevamente ejemplares de Lancaster y Heazlewood, obteniendo diagramas pobres con líneas difusas que tampoco pudieron indexarse. Estos datos están recogidos en la ficha 16-0164 del JCPDS. Desde entonces, en la bibliografía no han aparecido nuevos datos.

\section{Caracterización actual de la zaratita del Cabo Ortegal}

\section{Material y métodos experimentales}

El espécimen histórico analizado, zaratita del Cabo Ortegal, Zara-E-1, pertenece a la colección histórica de minerales del Museo Nacional de Ciencias Naturales de Madrid. Tiene un peso de 227,63 gy unas dimensiones de $7 \times 6 \times 3 \mathrm{~cm}$. El mineral es apenas una fina pátina de color verde esmeralda sobre una masa metálica de magnetita-hematites (Fig. 1). Pequeñas micro-partículas tomadas de la caja Zara-E-1 fueron incluidas en una resina epoxy y una vez pulida su superficie fueron estudiadas en microsonda electrónica (MSE) utilizando un equipo Jeol Superprobe

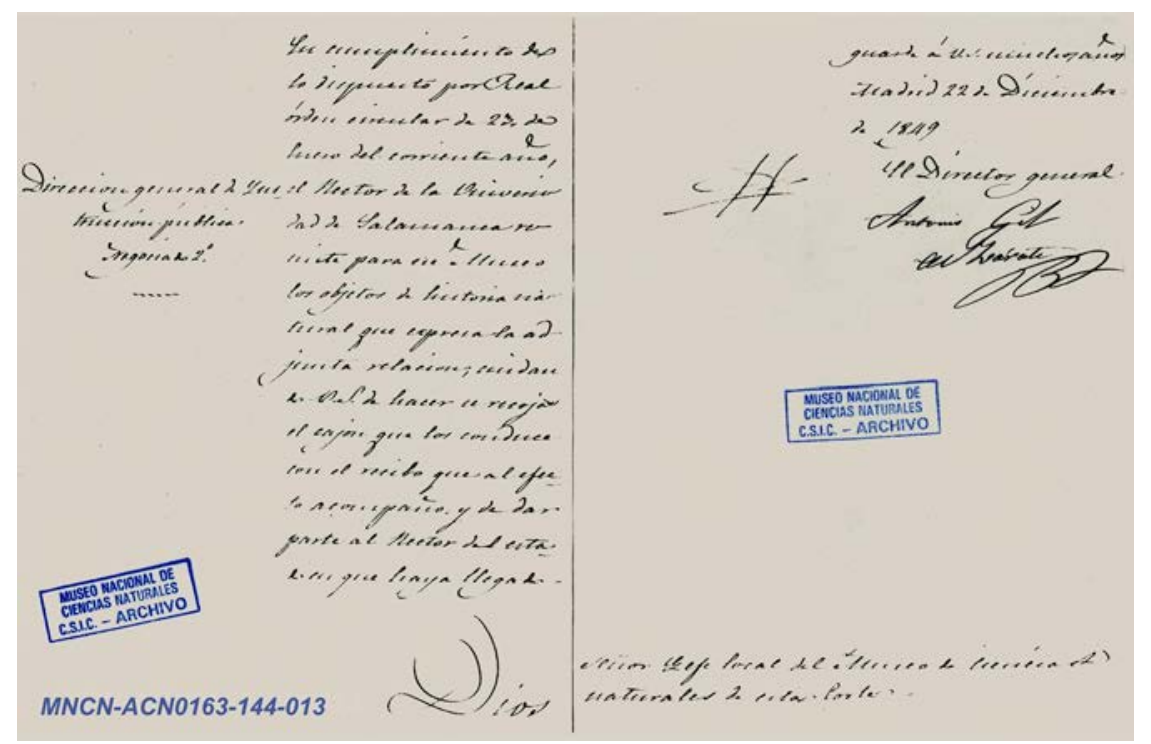

Fig. 2.-Texto de un legajo del Archivo Histórico del Museo con la llegada de las muestras de zaratita al Museo: "Dirección General de Instrucción Pública. Negociado 2. En cumplimiento de lo dispuesto por Real orden circular de 2 de Enero del corriente año, el Rector de la Universidad de Salamanca remite para el museo los objetos de Historia natural que expresa la adjunta relación cuidando Ud de hacer se recoja el cajón que los conduce con el recibo que al efecto se acompaña y de dar parte al Rector del estado en que haya llegado. Dios guarde a Ud muchos años. Madrid 22 Diciembre de 1849. El Director General Antonio Gil de Zarate. Sr. Jefe local del Museo de Ciencias Naturales de estos portes." 
JXA-8900M del Servicio de Microscopía Electrónica de la Universidad Complutense de Madrid. Las condiciones analíticas fueron: $20 \mathrm{KV}$ voltaje de aceleración, $50 \mathrm{nA}$ corriente de sonda, y $10 \mathrm{~s}$ de tiempo en pico y $5 \mathrm{~s}$ en cada background. Las medidas se han obtenido empleando un haz de diámetro comprendido entre 2 y $5 \mu \mathrm{m}$ y la muestra se recubrió con grafito. Se utilizaron los siguientes patrones y líneas: granate $(\mathrm{MnK} \alpha, \mathrm{FeK} \alpha)$, albita $(\mathrm{NaK} \alpha, \mathrm{AlK} \alpha)$, sillimanita $(\mathrm{SiK} \alpha)$, apatito $(\mathrm{CaK} \alpha, \mathrm{ClK} \alpha)$, níquel metal $(\mathrm{NiK} \alpha)$, galena $(\mathrm{SK} \alpha)$, cobalto metal $(\mathrm{CoK} \alpha)$, cobre metal $(\mathrm{CuK} \alpha)$, cromo metal $(\mathrm{CrK} \alpha)$. El carbono fue analizado previa metalización de la muestra con oro y utilizando un cristal LDE, escapolita $[\mathrm{CK} \alpha]$ como patrón, un voltaje de aceleración de $15 \mathrm{kV}$, una corriente de sondad de $20 \mathrm{nA}$ y un diámetro de haz de $10 \mu \mathrm{m}$. Los resultados fueron procesados utilizando un programa ZAF on-line. La muestra fue también estudiada, de manera no destructiva, en un microscopio electrónico de barrido ambiental (MEBA) provisto de (EDS) de FEI Impect Company donde se realizaron fotomicrografías y análisis químicos puntuales EDS. La difracción de rayos$\mathrm{X}$ (DRX) de las muestras se realizó en un equipo Phillips PW 1730/00 utilizando radiación $\mathrm{Cu} \mathrm{K \alpha}$, recogiendo los difractogramas entre 3 to $60^{\circ} 2 \theta$ con

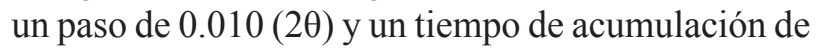
señal de 2 segundos. Los estudios de análisis térmico diferencial y termogravimetrico (ATD-TG) se realizaron en un equipo Setaram Labsys CS 32-CS 332 usando $5 \mathrm{mg}$ de muestra, crisoles de alúmina y una velocidad de calentamiento de $10^{\circ} \mathrm{C} \cdot$ minuto $^{-1}$. Los espectros de micro-Raman se realizaron en un microscopio Thermo-Fischer Raman con una resolución espacial de 1 micra. Se usó una fuente laser de $532 \mathrm{~nm}$ a $6 \mathrm{mw}$. La resolución espectral promedio fue $4 \mathrm{~cm}^{-1}$ y el tamaño del spot de $2 \mu \mathrm{m}$, utilizando el programa OMNIC 1.0. Los espectros de infrarrojo de las micro partículas de zaratita fueron recogidos en un microscopio Termo Scientific Nicolet iN10 FT-IR acoplado a un modulo Nicolet iZ10 FT-IR que tiene posibilidad de medir muestras de pocas micras, registrando los espectros con el programa OMNIC 1.0. Los estudios de catodoluminiscencia se realizaron con un equipo Gatam MonoCL acoplado a la cámara del microscopio MEBA antes descrito. Las condiciones de excitación del chorro de electrones para hacer las medidas de CL fueron de $25 \mathrm{kV}$.

\section{Resultados analíticos}

\section{Análisis químicos por MSE}

La tabla 1 muestra los resultados de 9 análisis puntuales realizados sobre las masas de zaratita mas limpias, previamente seleccionadas en el microscopio

Tabla 1-Análisis químicos por microsonda en diferentes puntos de Zaratita translúcida de color verde esmeralda (Cabo Ortegal, A Coruña)

\begin{tabular}{|c|c|c|c|c|c|c|c|c|c|}
\hline & 1 & 2 & 3 & 4 & 5 & 6 & 7 & 8 & 9 \\
\hline $\mathrm{CO}_{2}$ & 19,85 & 14,84 & 15,09 & 18,08 & 16,27 & 16,16 & 18,52 & 18,13 & 14,84 \\
\hline $\mathrm{SO}_{3}^{2}$ & 1,92 & 2,14 & 4,19 & 3,65 & 3,10 & 3,30 & 2,00 & 2,28 & 1,07 \\
\hline $\mathrm{P}_{2} \mathrm{O}_{5}^{3}$ & 0,00 & 0,00 & 0,00 & 0,00 & 0,01 & 0,00 & 0,00 & 0,00 & 0,00 \\
\hline $\mathrm{SiO}_{2}$ & 0,06 & 0,11 & 0,36 & 0,30 & 0,05 & 0,05 & 0,06 & 0,07 & 0,10 \\
\hline $\mathrm{Cl}^{-}{ }^{2}$ & 0,37 & 0,24 & 0,36 & 0,40 & 0,22 & 0,31 & 0,36 & 0,48 & 0,16 \\
\hline $\mathrm{NiO}$ & 67,07 & 69,61 & 69,67 & 66,74 & 69,06 & 70,65 & 69,00 & 68,63 & 70,66 \\
\hline $\mathrm{MgO}$ & 1,58 & 0,48 & 0,85 & 0,91 & 1,07 & 0,96 & 1,77 & 1,46 & 0,22 \\
\hline $\mathrm{FeO}$ & 0,74 & 0,59 & 0,06 & 0,08 & 0,00 & 0,16 & 0,58 & 0,27 & 0,75 \\
\hline $\mathrm{CoO}$ & 0,04 & 0,00 & 0,25 & 0,14 & 0,05 & 0,00 & 0,00 & 0,08 & 0,12 \\
\hline $\mathrm{CaO}$ & 0,24 & 0,10 & 0,04 & 0,05 & 0,05 & 0,05 & 0,21 & 0,23 & 0,09 \\
\hline $\mathrm{Al}_{2} \mathrm{O}_{3}$ & 0,17 & 0,01 & 0,02 & 0,02 & 0,01 & 0,04 & 0,05 & 0,00 & 0,04 \\
\hline $\mathrm{CuO}^{\circ}$ & 0,00 & 0,66 & 0,00 & 0,34 & 0,41 & 0,00 & 0,04 & 0,04 & 1,28 \\
\hline $\mathrm{MnO}$ & 0,02 & 0,54 & 2,03 & 1,54 & 0,04 & 0,01 & 0,05 & 0,02 & 0,14 \\
\hline Total & 92,07 & 89,33 & 92,91 & 92,24 & 90,34 & 91,69 & 92,64 & 91,68 & 89,47 \\
\hline $\mathrm{H}_{2} \mathrm{O}^{*}$ & 7,93 & 10,68 & 7,09 & 7,76 & 9,66 & 8,31 & 7,36 & 8,32 & 10,53 \\
\hline & \multicolumn{9}{|c|}{$\mathrm{H}_{2} \mathrm{O}^{*}$ Calculada por diferencia a 100} \\
\hline
\end{tabular}


óptico. A partir de estos datos se ha calculado la formula química del mineral empleando las siguientes consideraciones: (i) el contenido en agua se ha estimado por diferencia a100 del porcentaje total de óxidos, de este valor se calcula el contenido de grupos $\mathrm{OH}$ de la formula (ii) como los análisis se han realizado en zonas limpias y homogéneas de la muestra, sin inclusiones de otras fases distintas a la zaratita, todos los elementos químicos analizados se han incluido en la formula. Calculando en base a 7 oxígenos, según la fórmula ideal de la zaratita anhidra, $\mathrm{Ni}_{3} \mathrm{CO}_{3}(\mathrm{OH})_{4}$ obtenemos la siguiente formula promedio para la zaratita del Cabo Ortegal: $\mathrm{Ni}_{2.82} \mathrm{Mg}_{0.01} \mathrm{Fe}_{0.02} \mathrm{Cu}_{0.06}\left(\mathrm{CO}_{3}\right)_{1.17}\left(\mathrm{SO}_{4}\right)_{0.10}$ $(\mathrm{OH})_{2.90} \mathrm{Cl}_{0.01}$ Se trata de una fórmula mineralógica real obtenida a partir de los análisis químicos MSE de una muestra de muy baja cristalinidad, formada de forma natural capa a capa, con textura botriodal y de tamaños de partícula extremadamente pequeños bajo los umbrales de las ópticas electrónicas, en definitiva no es posible diferenciar fases mineralógicas.
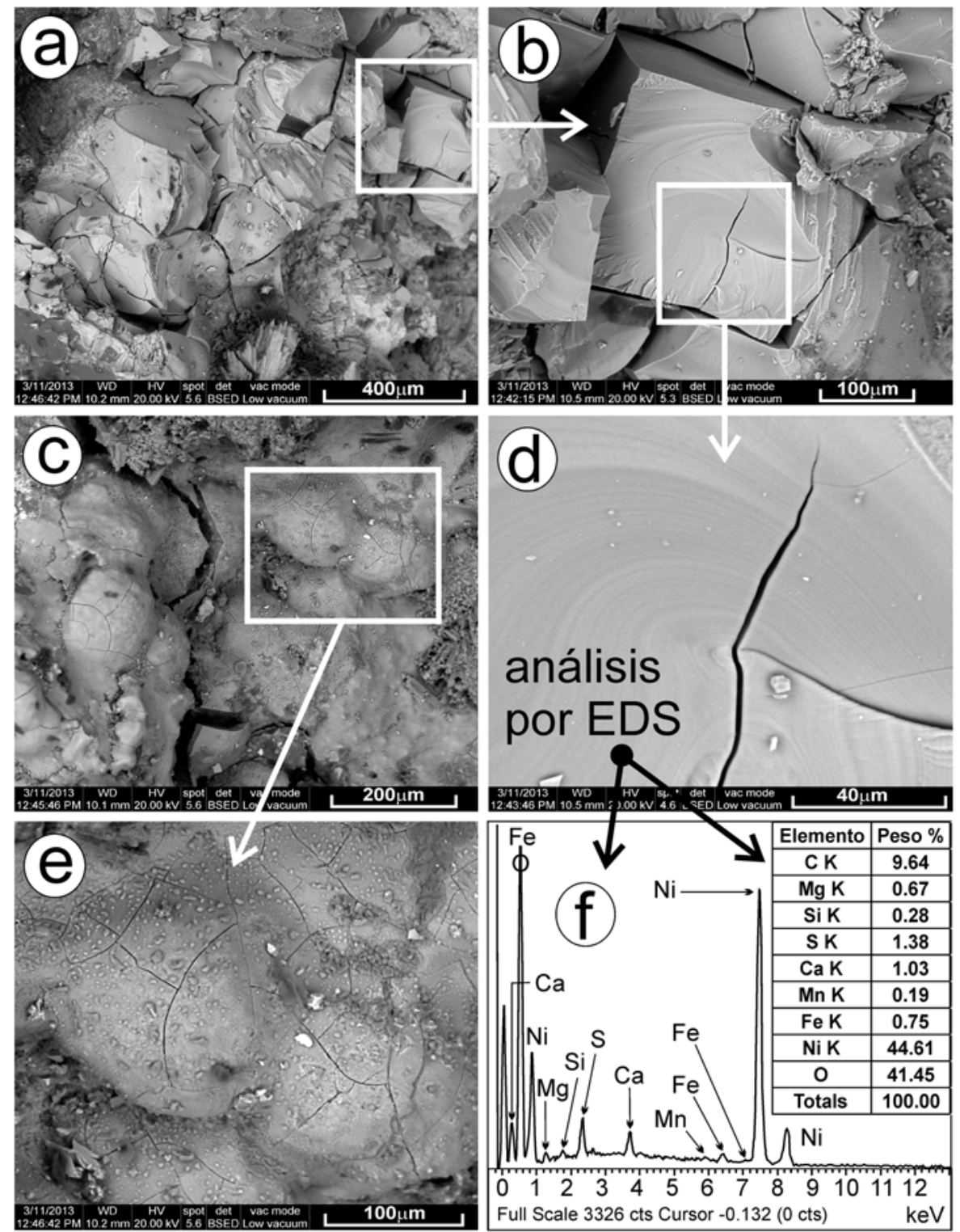

Fig. 3.-Fotografías de la muestra de zaratita bajo el microscopio electrónico de barrido y un ejemplo de análisis químico elemental por EDS de una de las zonas más puras de zaratita. Obsérvese su fractura concoidea, sus fisuras de deshidratación y su textura botrioidal. 


\section{Análisis de MEBA-EDS}

La figura 3a es una foto bajo el MEBA de la zaratita en gran parte (color blanco) pero incluyendo microcristales con variadas composiciones elementales bajo la sonda EDS de las que se deduce la siguiente mineralogía: (1) área rica en zaratita con magnesita y siderita y morenosita accesoria; (2) área rica en magnesita con zaratita y morenosita; (3) morenosita zaratita y magnesita; (4) zaratita con morenosita y magnesita y serpentina, (5) magnesita fibrosa con zaratita y morenosita. La figura $3 b$ es un detalle de la zaratita mostrando una fracturación concoidea en pseudocubos, junto con grietas de desecación por su constitución hidratada y posterior desecación. La figura $3 \mathrm{c}$ es otra parte de la zaratita que conserva su habito botrioidal característica de la deposición de minerales de baja cristalinidad en sistemas acuosos. La figura $3 \mathrm{~d}$ es un detalle de la zaratita mostrando las grietas de desecación y anillos concéntricos de las fracturas concoideas en materiales vítreos. La figura $3 \mathrm{e}$ es un detalle de los depósitos globulares botrioidales incluyendo todos ellos el sistema de fracturación producida por desecación. Finalmente la figura $3 \mathrm{f}$ incluye un espectro de composición elemental EDS tomado de una zona de zaratita muy limpia correspondiendo, obviamente, a un carbonato de níquel hidratado pero con elementos accesorios $\mathrm{Fe}, \mathrm{Mn}, \mathrm{Mg}, \mathrm{Si}, \mathrm{Ca}$ y $\mathrm{S}$ lo que no se puede evitar en compuestos de este tipo con baja cristalinidad y depositados capa a capa en texturas globulares.

\section{Difracción de Rayos X}

El diagrama de difracción de rayos $\mathrm{X}$ de la zaratita se presenta en la figura 4 a y corresponde a un sólido con un contenido superior al $80 \%$ de fase de baja cristalinidad, no aparecen picos correspondientes a reflexiones incluidas en la ficha 16-0164 del fichero JCPDS, y por supuesto, no pueden ser indexadas como una celdilla cubica. Las reflexiones de DRX oficiales 5.07(1) $\AA, 2.45(0.7) \AA$, 8.93(0.7) $\AA$ fueron obtenidas por Isaacs (1963) para una muestra de zaratita de Tasmania. En nuestro caso hemos utilizado zaratita-tipo de Cabo Ortegal utilizando con equipos de DRX digitales y no encontramos estas mismas reflexiones. Se han
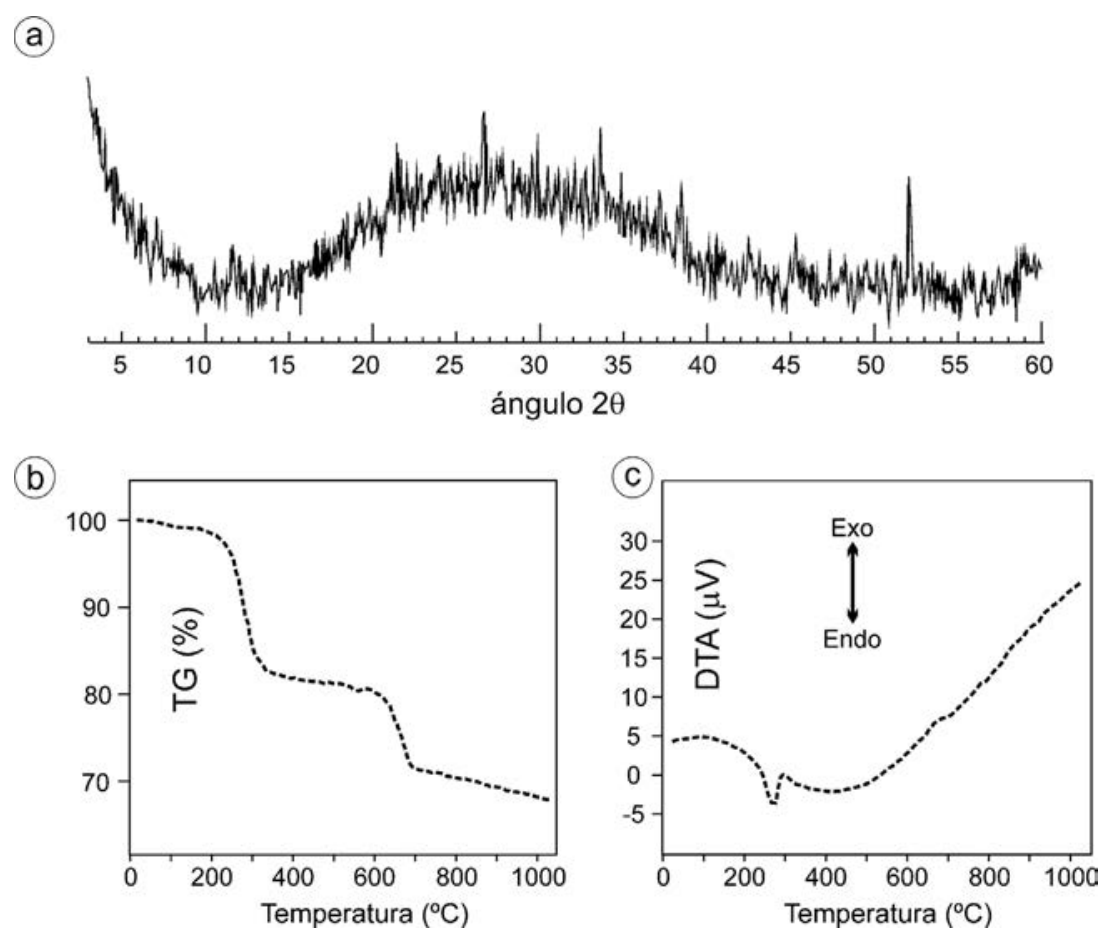

Fig. 4.-Análisis de la zaratita: (a) Difracción de rayos X mostrando estructura de baja cristalinidad (b) Termogravimetría y (c) Análisis térmico diferencial mostrando pérdidas de agua y de grupos hidroxilo. 
explorado los posibles minerales accesorios utilizando el programa español XPOWDER de Daniel Martín Ramos (Universidad de Granada), reduciendo la búsqueda a los siguientes elementos químicos: $\mathrm{Ni}, \mathrm{Fe}, \mathrm{Mn}, \mathrm{Ca}, \mathrm{Co}, \mathrm{Cu}, \mathrm{Si}, \mathrm{S}, \mathrm{Mg}, \mathrm{C}$ y $\mathrm{O}$, los pequeños picos pueden corresponder a las siguientes fases; magnesita ficha 86-2348 (pico a $2,759 \AA$ ), magnetita $86-246$ (pico a 2,548 , cove1lina 65-392 (pico a 4.050 $\AA$ ) compatibles con la paragénesis del Cabo Ortegal. La zaratita muestra un aspecto de pátina verde esmeralda sobre un bloque metálico masivo que también fue analizado por DRX. La composición mineralógica semicuantitativa de este bloque ha sido calculada con el programa XPOWDER que tambien cuantifica automáticamente el porcentaje de amorfos, obteniendo: $35 \%$ antigorita, $44 \%$ hematites, $18 \%$ magnetita y $3 \%$ de componentes de baja cristalinidad.

\section{Análisis térmicos ATD-TG}

En la figuras $4 \mathrm{~b}$ y $4 \mathrm{c}$ se presentan las curvas ATD y TG de la muestra Zara-E-1, calentada desde temperatura ambiente hasta $1000^{\circ} \mathrm{C}$. La curva termogravimétrica muestra una pequeña pérdida de peso del $0,98 \%$ entre los 25 y $120^{\circ} \mathrm{C}$, que puede ser atribuida a agua adsorbida por la muestra, ya que en ese rango de temperatura, la curva ATD no experimenta ningún cambio. A los $230^{\circ} \mathrm{C}$ comienza un proceso de pérdida de peso que culmina hacia los $650^{\circ} \mathrm{C}$ en el que se pierde un $19,23 \%$ del peso total y debe ser atribuido a la perdida de $\mathrm{H}_{2} \mathrm{O}$ y $\mathrm{CO}_{2}$ según: $\mathrm{NiCO}_{3}(\mathrm{OH})_{4}=$ $\mathrm{NiO}+\mathrm{CO}_{2}+2 \mathrm{H}_{2} \mathrm{O}$, como ha sido indicado por Fried y Dollimore (1994) y Shaheen (2002). El valor de la pérdida de peso obtenida por TG, $19,23 \%$, es compatible con los resultados de análisis químico de la muestra obtenidos por MSE. Hacia $\operatorname{los} 650^{\circ} \mathrm{C}$ en las curvas ATD y TG aparecen pequeñas alteraciones que pueden deberse a la presencia de impurezas (posiblemente magnesita) y a la recristalización del óxido de níquel (Rhamdhani et al., 2008).

\section{Espectrometría Raman}

El microscopio Raman DXR Thermofisher del MNCN dispone de un sistema óptico tradicional que permite observar micro-cristales de rocas, fotografiarlos y hacer el espectro Raman en zonas muy pequeñas de hasta 10 micras. Para casos como el que nos ocupa de la zaratita histórica es muy útil porque aporta análisis moleculares individualizados de los diferentes minerales de la muestra realizados de forma no destructiva. La determinación automática de fases mediante comparación de espectros Raman experimentales con otros de bases de datos minerales, por ejemplo RRUFF Arizona, junto con un conocimiento químico previo obtenido con el EDS del MEBA facilita la identificación de las fases minerales de la paragénesis de la zaratita de Cabo Ortegal (Fig. 5). Los bandas Raman observados a 941 y $982 \mathrm{~cm}^{-1}$ se producen por modos de deformación de aguas mientras que el pico $1073 \mathrm{~cm}^{-1}$ se puede asignar a vibraciones de tensión ע simétricas y $3 y$ antisimétricas del propio anión (CO3) ${ }^{2-}$ (Figura 5a-Zaratita) (Frost et al., 2008). La banda principal de la zaratita, hacia los $1366 \mathrm{~cm}^{-1}$, es debida a modos de vibración de tensión antisimétricos 3 עde aniones $(\mathrm{CO} 3)^{2-}$. La banda a $1609 \mathrm{~cm}^{-1}$ se atribuye a modos de enlaces de agua débilmente acopladas. Finalmente, el grupo de bandas a 2935, 2867 y 2753 $\mathrm{cm}^{-1}$ son atribuidos a moléculas orgánicas adsorbidas en la superficie mineral (Frost et al., 2008). La figura 5 b representa un espectro Raman realizado sobre una zona de minerales de color blanco. Destaca una banda de emisión Raman a $1115 \mathrm{~cm}^{-1}$ que corresponde con la banda máxima de la hidromagnesita. Esta fase coincide con la composición química previa elemental de $\mathrm{C}$ y $\mathrm{Mg}$ del EDS. El espectro de la figura 5c muestra el grupo de bandas a 227, 248, 284, 408, $495,611 \mathrm{~cm}^{-1}$ coincidiendo con el patrón RRUFF Hematite. En longitudes de onda superiores a los $1000 \mathrm{~cm}^{-1}$ el espectro muestra la típica banda ancha hacia los $1320 \mathrm{~cm}^{-1}$, característica de minerales de hierro, que podría corresponder mejor con una emisión foto-luminiscente que con una emisión Raman. Finalmente la figura $5 \mathrm{c}$ muestra tres grandes bandas a 233,446 y $608 \mathrm{~cm}^{-1}$ en la región baja del espectro coincidiendo perfectamente con la fase rutilo que es totalmente ajena a la paragénesis y se debe atribuir a una mancha blanca de Tipp-Ex del marcado institucional del propio museo. El complejo de pequeños $\mathrm{y}$ agudos picos en la región situada entre los $1000 \mathrm{y}$ los $1700 \mathrm{~cm}^{-1}$ es debido al polímero carbonado aglomerante del propio Tipp-Ex. 

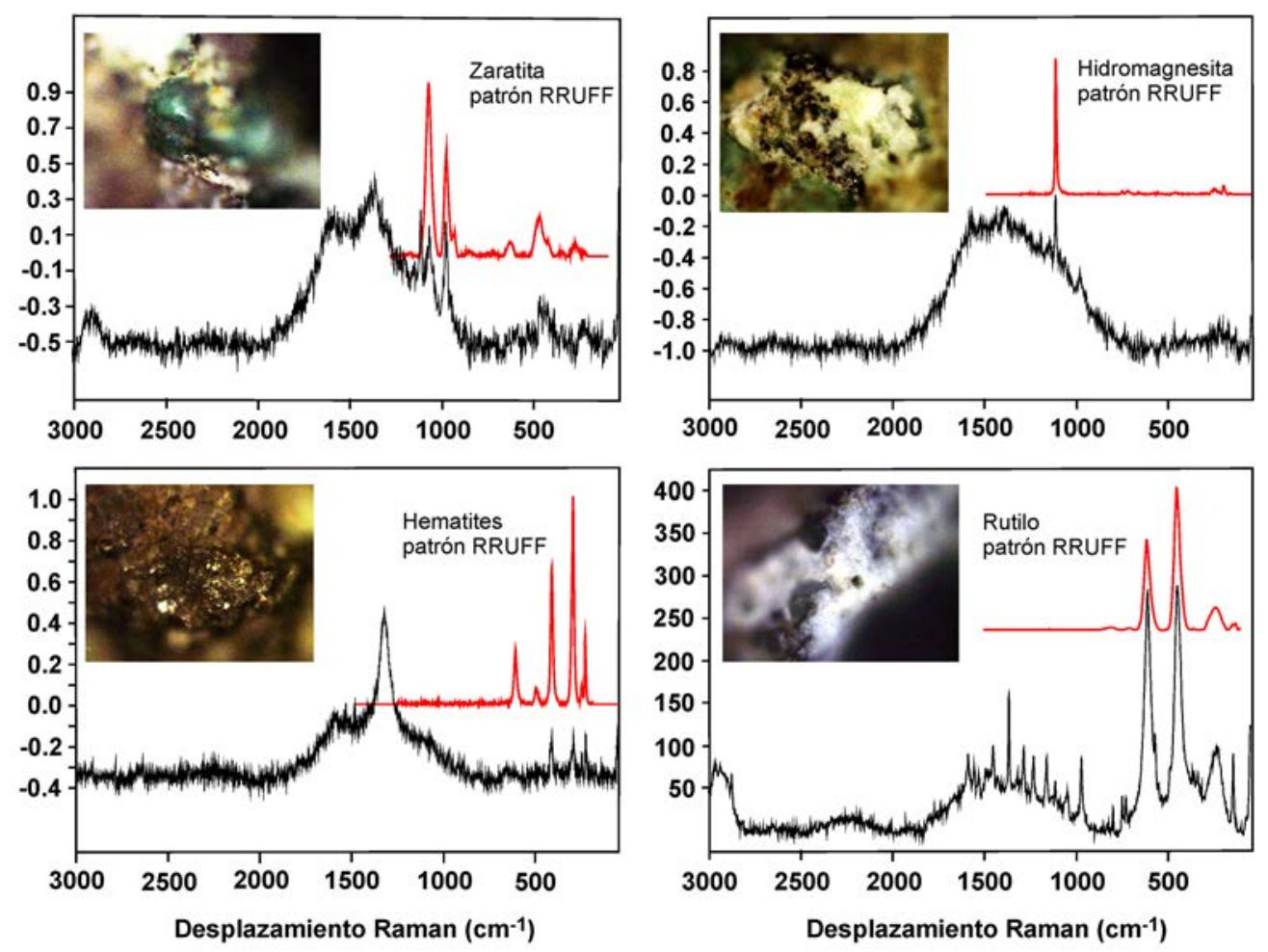

Fig. 5.-Espectros micro-Raman de diferentes fases minerales de la muestra de zaratita de Cabo Ortegal: (a) Zaratita, (b) Hidromagnesita, (c) Hematites, (d) Rutilo.

\section{Espectrometría IRTF}

La figura 6 muestra tres espectros IRTF correspondientes a: (a) Zaratita del condado de Texas (Pennsylvania, USA) realizado por Jones y Jackson (1993); (b) Zaratita de Cabo Ortegal medida por IRTF (KBr) en 1994 por Esperanza Menendez (Inst. Torroja, CSIC); (c) Zaratita de Cabo Ortegal medida por IRTF (ATR) en 2013 por Lola Gayo (Museo del Prado, Madrid). Se puede apreciar que a pesar de la variación de técnicas IRTF (ATR - KBr), separación temporal, diferentes equipos, técnicos, países y muestras de zaratita; en todos los casos existe bastante uniformidad de espectros centrados coincidiendo en los bandas a 767, 833, 1072, 1105, 1358, 1384 y $1575 \mathrm{~cm}^{-1}$. Los pequeños picos a 1575 y $1384 \mathrm{~cm}^{-1}$ se suelen interpretar como una doble composición de grupos hidroxilo y agua molecular siguiendo el clásico trabajo de Huang and Kerr (1960) para IRTF de carbonatos: (1) carbonatos hidratados sencillos con un doble pico destacado hacia los $1500 \mathrm{~cm}^{-1}$; (2) carbonatos con grupos hidroxilos o halógenos con dos bandas separados solo en su parte alta. La posibilidad de que se trate de nullaginita $\left(\mathrm{Ni}_{2}\left(\mathrm{CO}_{3}\right)(\mathrm{OH})_{2}\right)$ no es factible porque esta fase muestra un pico máximo a $1575 \mathrm{~cm}^{-1}$ con dos hombros acoplados a ambos lados del pico hacia los 1639 y los $1430 \mathrm{~cm}^{-1}$. Huang y Kerr (1960) afirman que existen lazos de unión entre el pico principal a $1575 \mathrm{~cm}^{-1}$ y los grupos $\mathrm{Ni}-\mathrm{CO}_{3}$ de acuerdo con los espectros IR de carbonatos anhidros romboédricos (grupo de la calcita) y rómbicos (grupo del aragonito) junto con las dos hombreras espectrales a 1639 y $1430 \mathrm{~cm}^{-1}$ que pueden ser atribuidas a grupos hidroxilos y a halógenos. Siguiendo las asignaciones de Frost et al. (2008), las bandas con máximos hacia los $1360 \mathrm{~cm}^{-1}$ y $1380 \mathrm{~cm}^{-1}$ pueden ser atribuidas a modos de vibración de tensión antisimétricos $\Downarrow 3$ de grupos $\left(\mathrm{CO}_{3}\right)^{2-}$. Las múltiples bandas observadas en el espectro IR indican una distorsión del anión $\left(\mathrm{CO}_{3}\right)^{2-}$ en la estructura de la Zaratita. La banda infrarroja de la zaratita a 1072 $\mathrm{cm}^{-1}$ puede ser asignada al modo de vibración de 


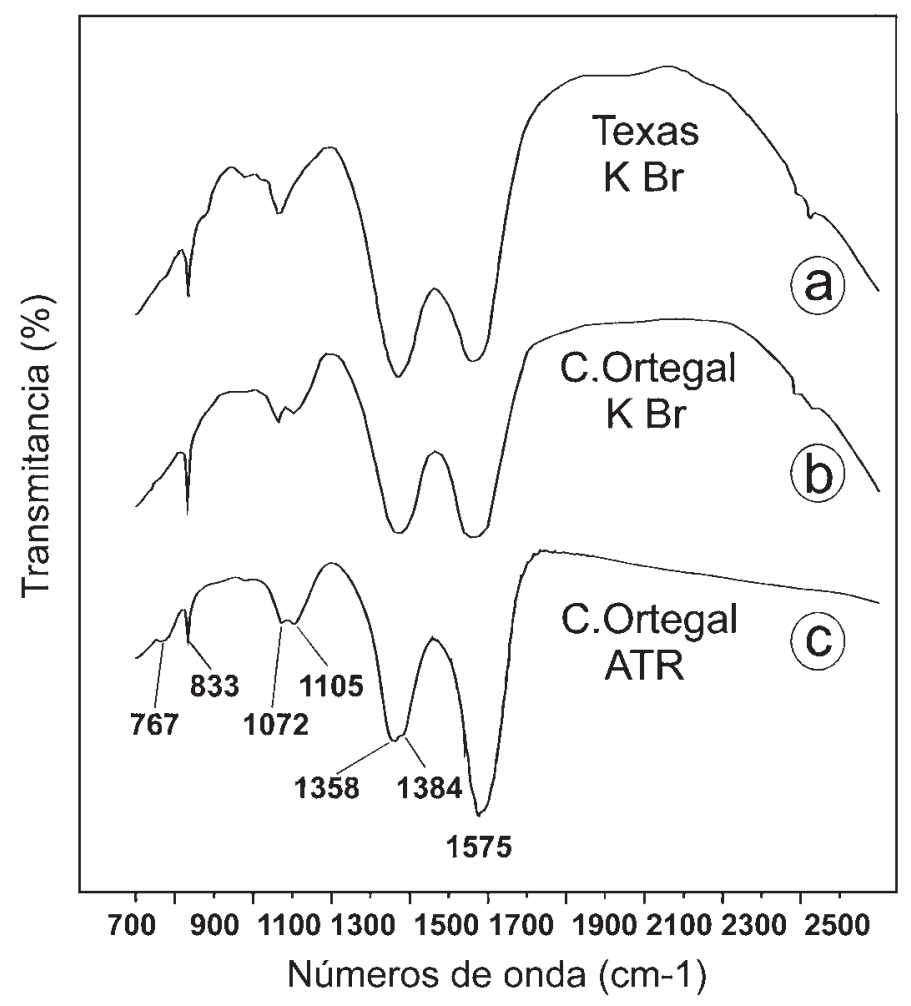

Fig. 6.-Espectros de FT-IR de zaratitas: (a) Texas County (Pennsylvania USA) en KBr, (b) Cabo Ortegal (La Coruña) en KBr en 1994; (c) Cabo Ortegal por ATR en 2013.

tensión simétrica ע correspondiente a vibraciones de deformación de grupos OH. El pico IR a $833 \mathrm{~cm}^{-1}$ se puede atribuir a modos de vibración de flexión 2 ע de la zaratita. Por todo esto, se puede decir que los espectros IR medidos en granos translúcidos de color verde-esmeralda de muestra tipo recogida en 1849 corresponden a zaratita propiamente dicha.

\section{Catodoluminiscencia $(\mathrm{CL})$}

La zaratita debería tener una emisión de luminiscencia de baja intensidad, por varios motivos: (i) presencia de níquel es un ión depresor de CL, (ii) contener casi un $1 \%$ de hierro, (iii) contener agua molecular y (iv) tratarse de un compuesto de baja cristalinidad. Sin embargo, la realidad analítica de la zaratita dentro del equipo ESEM-CL del Museo Nacional de Ciencias Naturales proporciona una emisión fotónica de CL de 25.000 unidades centrada en la banda espectral hacia los $610 \mathrm{~nm}$ (Fig. 7a). La muestra de zaratita, bajo ESEM a 5 micras, es mayoritariamente carbonato de níquel hidratado, pero con muy baja cristalinidad y con moderadas cantidades adicionales de $\mathrm{Fe}, \mathrm{Mg}, \mathrm{Ca}$ cercanas al $1 \%$ bajo la sonda EDS del ESEM. Por otra parte, algunos cationes divalentes como $\mathrm{Zn}, \mathrm{Co}, \mathrm{Ni}, \mathrm{Cu}, \mathrm{y}$ $\mathrm{Pb}$ sustituyen fácilmente al $\mathrm{Ca}$ en la estructura de la calcita, como se puede observar en experimentos de absorción de iones en calcita. El radio iónico del $\mathrm{Ni}^{2+}$ es $69 \mathrm{pm}$ en coordinación tetraédrica y de $83 \mathrm{pm}$ en coordinación octaédrica. Esto explicaría que el pico a $610 \mathrm{~nm}$ de la zaratita tuviera su origen en clústeres cristalográficos de $\mathrm{CaCO}_{3}$ sin poder determinar precisamente su estructura cristalina de aragonito o de calcita porque nuestras comparaciones experimentales con un aragonito violeta de Minglanilla (Fig. 7b) y con una calcita- blanca de Macael no permiten hacer comparaciones (Fig. 7c). En el caso del aragonito de Minglanilla por su elevado contenido en tierras raras y en el caso de la calcita por sus tensiones mecánicas. En todo caso, todos ellos muestran el pico antes descrito hacia los $400 \mathrm{~nm}$ relacionado 


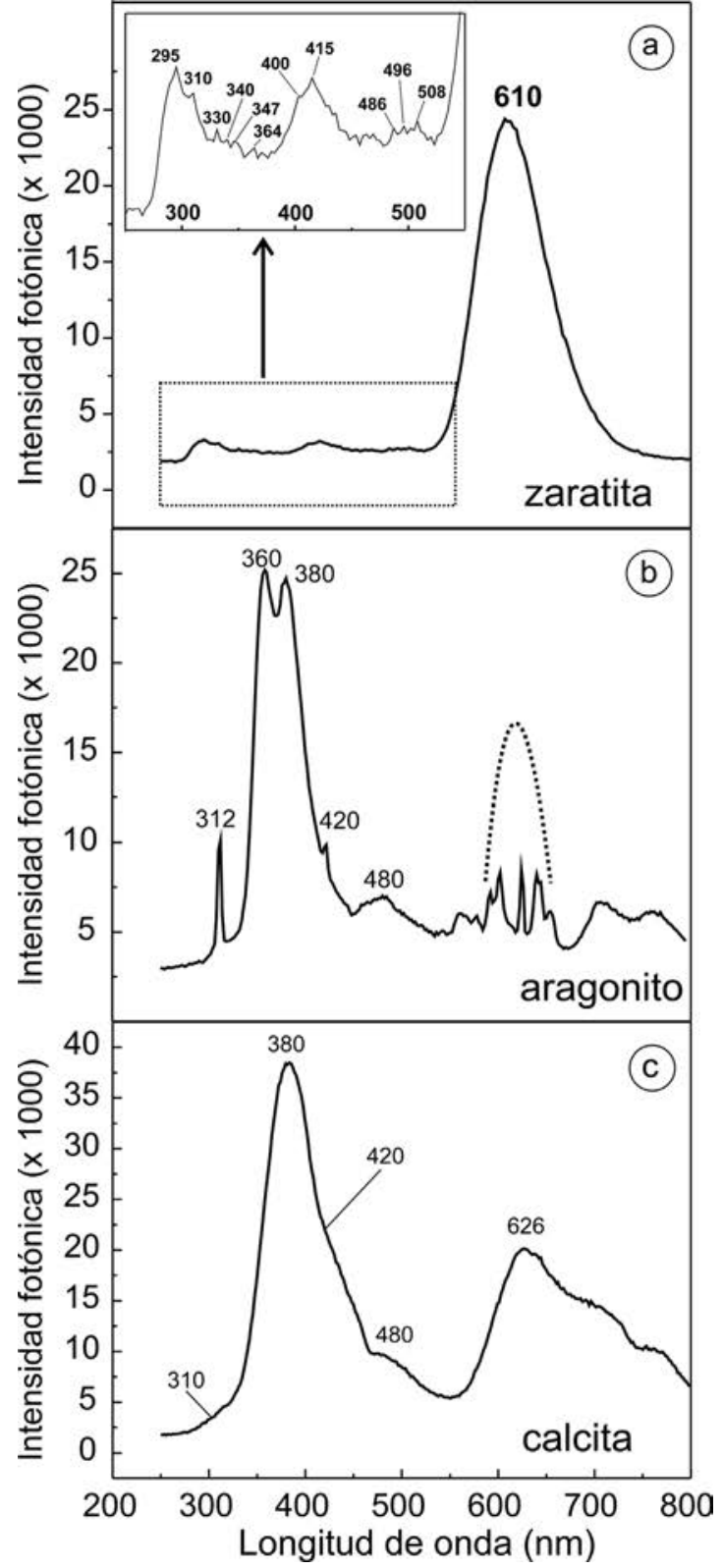

Fig. 7.-Espectros de catodoluminiscencia espectral medidos dentro del microscopio electrónico de barrido ambiental del Museo: (a) zaratita de Cabo Ortegal, (b) aragonito violeta de Minglanilla (Cuenca) con tierras raras, (c) Mármol blanco de Macael (Almería).

con defectos de electrones asociados a los grupos $\mathrm{CO}_{3}$ y algunas otras peculiaridades espectrales de las zaratitas más difíciles de explicar.

\section{Discusión}

Resulta curioso que Casares no publicara en una revista científica el descubrimiento de la zaratita y morenosita, limitándose a enviar a la Real Academia de Ciencias una pequeña memoria sobre su caracterización química donde revindica el descubrimiento. Agustín Martínez Alcibar, insigne Ingeniero de Minas, es el encargado de discutir los aspectos geológicos, mineralógicos y cristalográficos de ambas especies en sus dos artículos de 1850 y 1851 . Realmente, este último autor consiguió un gran éxito en sus publicaciones ya que fueron el vehículo para que las denominaciones de ambos minerales llegaran a ser adoptadas universalmente. Considerando además que Casares solo publicó artículos sobre análisis químicos de aguas, vinos y productos químicos y nunca sobre minerales (Cid, 2007), parece fundamentado considerar que este autor se limitó a estudiar las muestras en el laboratorio a petición del verdadero descubridor, posiblemente alguna persona con conocimientos de minería. Esta hipótesis está avalada por el siguiente párrafo que puede leerse al principio del articulo de 1850 de Martínez Alcibar: "considerando estas manchas verde esmeralda como indicaciones de mineral de cobre, se hizo en el año 1845 un registro, abriendo una pequeña labor." La caracterización química y estructural de la zaratita del Cabo Ortegal fue dificultosa debido a su baja cristalinidad. La propia composición de esta muestra, formada capa a capa, con texturas botriodales, con tamaños de partícula extremadamente pequeños, implica una falta de estequiometria y pequeñas diferencias en la composición química de ejemplares procedentes de otras localidades. Los datos de difracción de la zaratita publicados por Fenoglio en 1934(a) y, sobre todo, la ficha 16-0164 del JCPDS de 1966, obtenida con muestra de Tasmania definiendo al mineral como una fase estequiométrica con picos de DRX característicos, han supuesto un obstáculo más que una ayuda en los estudios de la zaratita-tipo. Los estudios de difracción de la muestra Zara-E-1 confirman que se trata de una fase de baja cristalinidad con impurezas de magnesita, magnetita, covellina y cuarzo compatibles con la paragénesis del Cabo Ortegal.

Los estudios de espectroscopia Raman e IRTF realizados en este trabajo caracterizan perfectamente a la zaratita del Cabo Ortegal. Las bandas del espectro Raman son similares a las obtenidas por Frost et al. (2008, 2010), en su estudio de la zaratita de 
Hazelwood, y el espectro IRTF del mineral corresponde al de zaratita por las dos bandas a $1577 \mathrm{y}$ $1370 \mathrm{~cm}^{-1}$ que la diferencian de la nullanginita, $\mathrm{Ni}_{2}\left(\mathrm{CO}_{3}\right)_{2}(\mathrm{OH})_{2}$, la cual presenta una única banda con máximo a $1546 \mathrm{~cm}^{-1}$ y dos hombros a $1639 \mathrm{y}$ $1430 \mathrm{~cm}^{-1}$ (Huang y Kerr, 1960).

El espectro Raman de la muestra Zara-E-1 indica la presencia de una pequeña proporción de agua débilmente ligada (banda de $1609 \mathrm{~cm}^{-1}$ ) y el de IRTF presenta pequeños bandas a 3652 y $3686 \mathrm{~cm}^{-1}$ que sugieren trazas de agua. La Curva TG indica una ligera pérdida de peso (inferior al $1 \%$ ) entre $25-120^{\circ} \mathrm{C}$ que puede deberse a agua adsorbida.

La emisión CL de los carbonatos en la región espectral del rojo-infrarrojo está asociada a la presencia o ausencia de iones activadores $\left(\mathrm{Mn}^{2+}\right.$, tierras $\operatorname{raras}^{2+/ 3+}$ ), de modificadores de la sensibilidad luminiscente $\left(\mathrm{Pb}^{2+}\right)$ o de depresores de luminiscencia $\left(\mathrm{Fe}^{2+/ 3+}, \mathrm{Ni}^{2+}, \mathrm{Co}^{2+}\right)$, siendo el ion $\mathrm{Mn}^{2+}$ el activador de emisión de luz más importante (Gotze, 2012). Además, muchos carbonatos suelen exhibir una banda de emisión de luminiscencia alrededor de los $400 \mathrm{~nm}$ probablemente relacionada con defectos de electrones asociados a los grupos $\mathrm{CO}_{3}$ (Habermann et al., 1997). El ión $\mathrm{Mn}^{2+}$ es un activador con orbitales $3 \mathrm{~d}$ externos parcialmente rellenos de electrones y por lo tanto sensibles a las fuerzas circundantes del campo eléctrico cristalino. Por lo tanto, la longitud de onda de las emisiones catodoluminiscentes asociadas a los iones $\mathrm{Mn}^{2+}$ dependen de la estructura cristalina; por ejemplo, en coordinación tetraédrica el ion $\mathrm{Mn}^{2+}$ proporciona emisión espectral de luz verde-amarilla mientras que en coordinación octaédrica lo hace en la región del rojo-anaranjado. La emisión CL activada por el ion $\mathrm{Mn}^{2+}$ en aragonito $\left(\mathrm{CaCO}_{3}\right.$, rómbico) tiene una banda espectral hacia los $580 \mathrm{~nm}$; en calcita $\left(\mathrm{CaCO}_{3}\right.$, trigonal) hacia los $620 \mathrm{~nm}$ y en magnesita $\left(\mathrm{MgCO}_{3}\right.$, trigonal) hacia los $655 \mathrm{~nm}$. (Calderón et al., 1996).

Las cuatro técnicas empleadas en la caracterización coinciden en señalar un bajo contenido de $\mathrm{H}_{2} \mathrm{O}$ en la muestra. Este resultado está avalado también por el análisis MSE que permite calcular la siguiente formula química para el mineral: $\mathrm{Ni}_{2,82} \mathrm{Mg}_{0,01} \mathrm{Fe}_{0,02} \mathrm{Cu}_{0.06}\left(\mathrm{CO}_{3}\right)_{1.17}\left(\mathrm{SO}_{4}\right)_{0.10}(\mathrm{OH})_{2.90} \mathrm{Cl}_{0,01}$, a la que faltan una cierta cantidad de $\mathrm{Ni}$, OH y 4 grupos $\mathrm{H}_{2} \mathrm{O}$ para ser similar a la propuesta por Smith y Brush (1853) para la zaratita americana: $\mathrm{Ni}_{3} \mathrm{CO}_{3}(\mathrm{OH})_{4} 4 \mathrm{H}_{2} \mathrm{O}$. En 1850, en el húmedo ambiente del Cabo Ortegal el mineral tuvo mayor contenido en $\mathrm{H}_{2} \mathrm{O}$, lo que está de acuerdo con la observación de Casares: "calentado este mineral en el tubo cerrado por un extremo da vapores acuosos". Hoy día, después de 150 años de almacenaje en un ambiente tan seco como el de Madrid, está justificada la pérdida total del agua de hidratación.

\section{Conclusiones}

Los análisis de difracción de rayos-X del ejemplar histórico de zaratita depositado en el MNCN, indican que se trata de un material de baja cristalinidad. Los nueve análisis químicos realizados por MSE del ejemplar depositado en el MNCN indican una distribución homogénea de los elementos mayoritarios y una relación molar $\mathrm{Ni} / \mathrm{CO}_{3}$ próxima a la de la bibliografía.

En el descubrimiento de la zaratita Casares debió actuar a petición de otra persona, posiblemente un práctico en minería, quien reconoció la importancia de las manchas verde esmeralda en el acantilado y recogió el mineral para su análisis.

Los estudios de espectroscopia infrarroja y Raman demuestran que el ejemplar estudiado contiene poco agua de hidratación.

La zaratita debería tener muy baja luminiscencia porque el níquel es un ión depresor de CL, por contener casi un $1 \%$ de hierro, por contener teórica agua molecular y por tratarse de un compuesto de baja cristalinidad. Sin embargo, tiene un potente pico CL a $610 \mathrm{~nm}$ por posibles clústeres de Ca-Mn y una pequeña luminiscencia UV-azul intrínseca atribuible a defectos de oxígeno y tensiones estructurales.

Las diferencias en cuanto a la ausencia de agua de hidratación del ejemplar del MNCN pueden deberse al almacenaje, durante 150 años, en un ambiente tan seco como el de Madrid

\section{Referencias}

Calderón, T.; Towsend, P; Beneitez, P; Garcia-Guinea, J; Millán, A.; Rendell, H.M.; Tookey, M.; Urbina, M. \& Wood, R. (1996). Crystal field effects on the Thermoluminescence of manganese in carbonate lattices. 
Radiation Measurements, 26: 719-731. http://dx.doi. org/10.1016/S1350-4487(97)82886-7

Cid, R. (2007). A vida e a obra de Antonio Casares Rodríguez. http://antoniocasares.blogspot.com.es.

Dana, E.S. (1878). A Text-Book of Mineralogy. John Wiley and Sons. New York. Second edition. 388 pp.

Fenoglio, M. (1934a). Sulla structura cristallina della Zaratite. Periodico di Mineralogía, 5: 33-36.

Fenoglio, M. (1934b). Nuove riherche sulla Zaratite: Zaratita sintetica. Periodico di Mineralogía, 5: 265-274.

Fried, D. \& Dollimore, D. (1994). Isothermal and rising temperature kinetic studies of doped non-stoichiometric basic nickel carbonate. Journal of Thermal Analysis, 41: 323-336. http://dx.doi.org/10.1007/ BF02549318

Frost, R.L.; Dickfos, M.J. \& Jagannadha-Reddy, B. (2008). Raman spectroscopy of hydroxy nickel carbonate minerals nullaginite and Zaratite Journal of Raman Spectroscopy, 39: 1250-1256. http://dx.doi. org/10.1002/jrs.1978

Frost, R.L.; Keeffe, E.C. \& Reddy, B.J. (2010). Characterisation of Ni carbonate-bearing minerals by UV-Vis-NIR spectroscopy. Transition Metal Chemistry, 35(3): 279287. http://dx.doi.org/10.1007/s11243-009-9324-7

Gotze, J. (2012). Application of Cathodoluminescence Microscopy and Spectroscopy in Geosciences. Microscopy and Microanalysis, 18: 1270-1284. http://dx.doi.org/10.1017/S1431927612001122

Habermann, D.; Götze, J.; Neuser, R. \& Richter, D.K. (1997). The phenomenon of intrinsic cathodoluminescence: Case studies of quartz, calcite and apatite. Zentralblat für Geologie und Paläontologie Teil 1, Heft 10-12: 1275-1284.

Huang, C.K. \& Kerr, P.F. (1960). Infrared study of the carbonate minerals. American Mineralogist, 45: 311-324.

Isaacs, T.(1963). The mineralogy and chemistry of the nickel carbonates. Mineralogical Magazine, 33: 663-678. http://dx.doi.org/10.1180/minmag.1963.033.263.04

Jones, G.C. \& Jackson, B. (1993). Infrared transmission spectra of carbonate minerals. Ed. Chapman \& Hall. London, $256 \mathrm{pp}$.
Kiselev, A.E. (1938). Annals of the Institute of Mines, Leningrad, vol. II, p. I. Abstr. in Mineralogical Magazine. 9: 267.

Martinez Alcibar, A. (1850). Raro e importante mineral de níquel. Revista Minera, 1: 302-306.

Martinez Alcibar, A. (1851). Sobre el mineral de nickel de Galicia, con algunas cosideraciones sobre el polimorfismo del sulfato de nickel y de otras sustancias. Revista Minera, 2: 175-190.

Palache, C.; Berman, H. \& Frondel, C. (1951). The system of Mineralogy of James Dwight Dana and Edward Salisbury Dana, Yale University 1837-1892, Volume II. John Wiley and Sons, Inc., New York, 7th edition, 245-246 pp.

Petterd, W.F. (1894). A catalogue of minerals known to occur in Tasmania, with notes on their distribution. Paper and Procceding of Royal Society of Tasmania for 1893, 1-72.

Rhamdhani, M.A.; Jak, E. \& Hayes, P.C. (2008). Basic nickel carbonate: Part II. Microstructure evolution during industrial nickel production from basic nickel carbonate. Metallurgical and Materials Transactions B, 39(2): 234-45. http://dx.doi.org/10.1007/ s11663-008-9139-5

Shaheen, W.M. (2002). Thermal behavior of pure and binary basic nickel carbonate and ammonium molybdate systems. Materials Letters, 52: 272-82. http:// dx.doi.org/10.1016/S0167-577X(01)00406-2

Silliman, B. (1847). Hydrate of Nickel a New Mineral. American Journal of Science, 3: 407-409.

Silliman, B. (1848). On Emerald of Nickel from Texas, Lancaster County, Pa. American Journal of Science, 6: 246-249.

Smith, J.L. \& Brush, G.J. (1853). Reexamination of American Minerals. Part II- Chesterlite; Loxoclase; Danbury Feldspars; Haddan Albite; Greenwood Mica; Biotite; Magarodite; Chesterlite Talc; Rhodophillite; Cumnmigtonite; Hydrous Anthophillite; Monrolite; Ozarkite; Dysyntribite; Gibbsite; Emerald Nickel. American Journal of Science, 16: 41-53.

Williams, K.L.; Threadgold, I.M. \& Hounslow, A.W. (1959). Hellyerite, a new nickel carbonate from Heazlewood, Tasmania. American Mineralogist, 44: 533-538. 